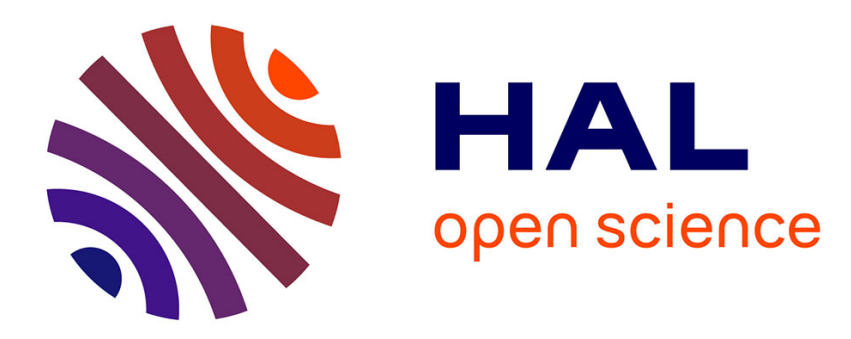

\title{
Accelerator and new accelerating schemes
}

B. Heusch, G. Matthieussent

\section{- To cite this version:}

B. Heusch, G. Matthieussent. Accelerator and new accelerating schemes. Revue de Physique Appliquée, 1988, 23 (9), pp.1420-1421. 10.1051/rphysap:019880023090142000 . jpa-00245965

\section{HAL Id: jpa-00245965 https://hal.science/jpa-00245965}

Submitted on 1 Jan 1988

HAL is a multi-disciplinary open access archive for the deposit and dissemination of scientific research documents, whether they are published or not. The documents may come from teaching and research institutions in France or abroad, or from public or private research centers.
L'archive ouverte pluridisciplinaire HAL, est destinée au dépôt et à la diffusion de documents scientifiques de niveau recherche, publiés ou non, émanant des établissements d'enseignement et de recherche français ou étrangers, des laboratoires publics ou privés. 


\title{
Accelerator and new accelerating schemes
}

\author{
B. Heusch $\left({ }^{1}\right)$ and G. Matthieussent $\left({ }^{2}\right)$ \\ (1) Centre de Recherches Nucléaires, Strasbourg, France \\ (2) CNRS, Laboratoire de Physique des Gaz et des Plasmas, Orsay, France
}

Recent research and progress in particle accelerators for high energy physics has been the subject of a meeting held, on July 1987, in Strasbourg, during the General Conference of the French Physical Society [1]. This issue of the Revue de Physique Appliquée, without being a detailed account of the meeting, contains most of the papers which have been presented.

About fifty physicists, working in nuclear and high energy physics, astrophysics and plasma physics had the opportunity to discuss accelerators under construction as well as the novel acceleration techniques which have appeared during the past few years. Beyond the exchange of information between physicists from different disciplines, this meeting has exposed the fruitful collaboration of various groups working on novel accelerator schemes using plasmas. In particular, this meeting resulted in a clearer definition of the main requirements for the energy and luminosity of such accelerators.

In order to study the window of existence of the Higg's boson, the structure of leptons and quarks as well as other outstanding questions in high energy physics [2], it is desirable to develop linear collider with energy $>10 \mathrm{TeV}$. Although the energy of accelerators has been increased by five order of magnitude during the last fifty years, and the cost per $\mathrm{MeV}$ has been reduced at nearly the same rate, the devices under design (S.S.C, L.H.C), to be completed in ten or twenty years, are only extrapolation of actual accelerators. Their accelerating electric fields are of the order of a few tens of $\mathrm{MV} / \mathrm{m}$, technical improvements (supraconductive cavities, short wave lengths) are not expected to allow electric fields $>100 \mathrm{MV} / \mathrm{m}$.

The astonishing increase in the energy of particles delivered by accelerators has been obtained mainly through the application of novel accelerating schemes rather than improvement of existing technologies. Thus, along similar lines, novel accelerator schemes using plasmas $[3,4]$ have recently been proposed as a consequence of recent progress in high power lasers [5] and high current and low energy accelerators. Why use plasmas? In usual accelerators, the main limitation of the electric field comes from breakdown near the surface of the wave guide ; the use of a fully ionized plasma as a support for the longitudinal accelerating electric field removes this limitation.

Electrostatic plasma waves can be excited in this medium by two different resonant processes with longitudinal electric fields of the order of $10 \mathrm{GV} / \mathrm{m}$. The first scheme uses the beatwave excited in a plasma by two intense laser beams (few Joules, tens of picosecond) while the second scheme relies on the wake field excited behind a relativistic electron beam (few $\mathrm{MeV}$, hundred of $\mathrm{kA}$ during nanoseconds) [4]. These two novel accelerating techniques are tested in laboratories in order to prove the principles of the new mechanisms involved. For the plasma beatwave scheme, experiments $[5,6]$ are presently conducted on homogeneous plasmas with densities of the order of $10^{17} \mathrm{~cm}^{-3}$ and millimeters in length, where longitudinal electric fields as high as $1 \mathrm{GV} / \mathrm{m}$ have been measured. Electron injection and acceleration, on a $\mathrm{MeV}$ scale, are now being tested.

In order to reach particle energies of tens of $\mathrm{TeV}$, an accelerator based on one or the other of these novel techniques [7], laser beat-waves or wake fields, would require staging ; many unknowns remain, concerning luminosity, beam energy dispersion, power efficiency, all are parameters which are function of the synchronisation between stages and of the way the laser beams are applied to each stage. The aim of actual experiments is just to provide the physical basis on which a new generation of high energy accelerators could rely [8].

In nuclear physics, progress proceeds mainly from the study of the induced reactions by the impact of heavy ions on nuclei : while keeping the selectivity of excitation by light particles, heavy ions bring to the system under study a greater linear angular momentum. Nuclear matter can thus be obtained at the limit of its stability. New results have been obtained concerning the existence of isotopes far from stability, the limits of nuclear fusion, induced fission on nearly all nuclei, the shape of those nuclei, nuclear fragmentation and sequential processes [9].

Between 10 and $100 \mathrm{MeV} / \mathrm{n}$, the collisions of atomic nuclei show the transition from a regime where the mean nuclear field dominates (energy less 
than $20 \mathrm{MeV} / \mathrm{n}$ ) to another due to the interactions between nucleons when the energy exceeds the Fermi's energy in the nucleus (around $35 \mathrm{MeV} / \mathrm{n}$ ).

The Van de Graff, tandem accelerator of $35 \mathrm{MeV}$, Vivitron [10], being finished at Strasbourg, is the first of a new generation of electrostatic devices ; its original design minimizes electrical breakdown by a better distribution of the stored electrical energy. To be completed in 1990, this device will give multicharged ions with $20 \mathrm{MeV} / \mathrm{n}$ and $5 \mathrm{MeV} / \mathrm{n}$ for light and heavy ions, respectively, with a current of about $100 \mathrm{nA}$. The Vivitron is also characterized by the ability one can change the nature and energy of the accelerated ions. Such devices can be used as an injector for post acceleration; such an arrangement is presently used at Oak-Ridge, Stony Brook, Chalk River and Berlin, or is under construction at Daresbury, Catane and Saclay. At Saclay, a 9 MV tandem is coupled to an accelerator [11] made of resonant supraconductive cavities with an electric field of $2 \mathrm{MV} / \mathrm{m}$. However the energy of heavy ions is limited to $10,15 \mathrm{MeV} / \mathrm{n}$. With the coupling of a tandem to a cyclotron, either at ordinary temperature (Berlin) or with supraconductive materials (Chalk-River, Catane), the limit is of the order of $50 \mathrm{MeV} / \mathrm{n}$.

For high energies, 10 or $100 \mathrm{MeV} / \mathrm{n}$, cyclotrons or associations of them, supraconductor (AGOR, M.S.U.) or not (SARA, GANIL) are used. In some cases, resonant cavities are associated together (M.S.U., G.S.I., Darmstadt). Finaly for energies greater than $100 \mathrm{MeV} / \mathrm{n}$ synchrotrons are the only suitable devices: Saturne at Saclay with $1 \mathrm{GeV} / \mathrm{n}$ and S.I.S. at Darmstadt with $2 \mathrm{GeV} / \mathrm{n}$. At this last place a storage ring is in completion [12].

Beyond nuclear physics, high energy beams of heavy ions are planned to be used in inertial confinement fusion [13] : one expects to compress a mixture of deuterium and tritium enclosed in microballoons to 1000 times the solid density in order to initiate nuclear fusion. rections. The energies of the heavy ions, i.e. $\mathrm{B}_{\mathrm{i}}^{+}$, involved in such schemes are of the order of $10 \mathrm{GeV}$ with currents of the order of $10 \mathrm{kA}$. These characteristic values of energy and current could be obtained by simultaneous use of R. F. accelerators, linacs and storage rings. However, the comparison between the required beam parameters for heavy ion fusion and these obtained on actual devices, are a clear evidence of the effort to do.

The papers published in this special issue cover three subjects : conventional acceleration, plasmabased acceleration and acceleration processes in astrophysics. The first part of this volume is devoted to classical acceleration schemes for electrons and ions : review papers on devices and techniques, and a description of the Vivitron device. In the second part, the novel ideas using plasmas and laser beams are treated. Experiments in Canada (INRS-E) on beat-waves in laser-plasmas and at the Ecole Polytechnique on forward Raman scattering are the subject of two articles. The third part covers the acceleration processes in astrophysics. These processes are, most often, due to quasiperpendicular shock waves in the stellar medium in which Fermi mechanism accelerates particles to $\mathrm{GeV}$ energies.

\section{Acknowledgments.}

The associated editors, B. Heusch and G. Matthieusent thank the authors, the referres and the editorial staff and all those who participate in the edition of this special issue. 